\title{
Modelos de rehabilitación para la intervención del desorden músculo-esquelético en trabajadores
}

\author{
Rehabilitation models for intervention of \\ work-related musculoskeletal disorders
}

María C Trillos $\mathrm{Ch}^{1}$, Ingrid A Tolosa $\mathrm{G}^{1}$, Raquel Y Escobar $\mathrm{F}^{1}$

Forma de citar: Trillos MC, Tolosa IA, Escobar RY. Modelos de rehabilitación para la intervención del desorden músculoesquelético en trabajadores. Rev Univ Ind Santander Salud. 2016; (4): 536-547. DOI: http://dx.doi.org/10.18273/revsal. v48n4-2016012 (c) (i)

\section{RESUMEN}

Introducción: Los modelos en salud promueven el desarrollo de intervenciones para solucionar diferentes problemáticas poblacionales. Objetivo: El objetivo de este artículo es establecer un diagnóstico de los modelos existentes para la rehabilitación del desorden músculo esquelético (DME) en la población trabajadora, a través de una revisión documental y de la literatura científica disponible. Metodología: Se realizó una revisión sistemática de la literatura en las bases de datos Ovid SP, EBSCO HOST, PROQUEST, MEDLINE, Science Direct, PubMed, y otros motores de búsqueda, siguiendo las etapas de identificación, tamización, elegibilidad e inclusión consignadas en la guía PRISMA. Resultados: Se seleccionaron 72 artículos, de los cuales 60 documentos fueron tenidos en cuenta para el análisis bibliométrico; se identificaron y abordaron doce modelos teóricos del proceso salud enfermedad, los cuales mostraron un enfoque en rehabilitación clínica. Discusión y conclusiones: Se concluyó que los modelos de rehabilitación encontrados contienen diversos elementos que los hacen complementarios y que no generan conflicto entre ellos, por el contrario de acuerdo a las disciplinas que lo definen y la interdisciplinariedad que puedan tener, los hacen aún más sólidos en sus premisas para la rehabilitación de los (DME) en trabajadores. Adicionalmente se observa como los enfoques de los modelos de rehabilitación más recientes están dirigidos a fomentar las condiciones protectoras del trabajador con el fin de prevenir alteraciones en su condición de salud.

Palabras clave: Musculo esquelético, medicina física y rehabilitación, modelos en salud, trabajadores (DeCS).

1. Universidad del Rosario. Bogotá, Colombia.

Correspondencia: María C Trillos Ch. Dirección: Carrera 24 Nº3C-69. Bogotá, Colombia. Correo electrónico: maria.trillos@urosario.edu.co Teléfono: (571) 2970200 ext 3463. 
ABSTRACT

Introduction: Health models promote the development of interventions to address various population issues. Objective: The aim of this article is to establish a diagnosis of existing rehabilitation of musculoskeletal disorder in the working population models, through a literature review and the available scientific literature. Methodology: A systematic literature review was made in databases Ovid SP, EBSCO HOST, PROQUEST, MEDLINE, Science Direct, PubMed, among other research engines following the stages of identification, screening, eligibility and inclusion contained in the PRISMA guideline. Results: 72 articles were selected, of which 60 papers were considered for the bibliometric analysis; it they identified and addressed twelve theoretical models of health-disease process, which showed a focus in Clinical Rehabilitation. Discussion and conclusions: It was concluded that models rehabilitation found contain various elements that make them complementary and not create conflict between them, on the contrary according to the disciplines that define and interdisciplinary they may have, make them even stronger in their premises for rehabilitation of DME workers. Additionally it is seen as approaches most recent models are aimed at promoting rehabilitation protective worker conditions in order to prevent changes in their health condition.

Keywords: Musculoskeletal disorders, physical and rehabilitation medicine, health models, workers (MeSH).

\section{INTRODUCCIÓN}

Los modelos en salud han sido diseñados con el fin de promover el desarrollo de intervenciones para solucionar problemas dentro del proceso de salud enfermedad en la población. Así mismo son considerados una herramienta organizada, diseñada para categorizar ideas y enfoques que le dan sentido a fenómenos complejos ${ }^{1}$ en este caso en la salud del individuo y sus colectivos.

A lo largo del tiempo se han identificado modelos que datan de la edad media y que consideraban al individuo como un ser netamente pasivo receptivo frente a su salud hasta enfoques como el propuesto por el modelo Forense $^{2}$ en donde se tenía en cuenta las interacciones, conocimientos y motivaciones del ser humano o el modelo ecológico en el que se rescataba la influencia de los patrones culturales, las tendencias políticas, los sistemas económicos y las creencias frente a la condición de discapacidad ${ }^{3}$.

Con el paso del tiempo diferentes tendencias se han generado frente al tema de salud-enfermedad derivadas de los avances tecnológicos y la creciente atomización del proceso social, dada por la creación de múltiples especialidades en las diferentes disciplinas ${ }^{2}$. Lo que ha llevado a que se haya perdido la visión integral del ser humano y el contexto en donde se desempeña social y laboralmente.

Los modelos de rehabilitación para la intervención de los Desorden Músculo Esqueléticos (DME) relacionados con el trabajo, han sido desarrollados a partir de los modelos de salud. En algunos casos se han convertido en enfoques descontextualizados de las realidades sociales y laborales, razón por la cual se generan limitaciones en torno a la identificación de los múltiples factores desencadenantes de esta condición.

Es a partir de la aparición del modelo biopsicosocial que se reconoce nuevamente la importancia de una visión integral del individuo. Al articular al modelo social el modelo médico para explicar la condición de discapacidad y el retorno al trabajo mediante la comprensión del individuo, los sistemas involucrados y sus interacciones ${ }^{4-7}$.

La necesidad de obtener unos mejores resultados dentro de los procesos de rehabilitación ha llevado a generar programas de intervención a partir de algunos modelos en salud que facilitan el abordaje del equipo rehabilitador al trabajador.

Esta revisión presenta los diferentes modelos en salud, y específicamente los modelos en rehabilitación que se han desarrollado a través del tiempo y describe los alcances y limitaciones de cada uno de ellos a partir de sus características frente al abordaje en el contexto laboral. Con el fin de proveer el sustento teórico para el diseño de modelos de rehabilitación frente al DME que garantice la salud de los trabajadores.

\section{METODOLOGÍA}

Se realizó una revisión sistemática de la literatura siguiendo las etapas de identificación, tamizaje, elegibilidad e inclusión consignadas en la guía PRISMA (Preferred Reporting Items for Systematic reviews and Meta-Analyses) $)^{8}$. En la estrategia de búsqueda se emplearon los términos "Enfermedades 
Musculoesqueléticas", "Musculoskeletal Diseases", "Modelos teóricos", "Theoretical models" y "Rehabilitación”, "Rehabilitation”, cada uno de los conceptos se interseccionaron con la conjunción booleana "AND".

La búsqueda se realizó en bases de datos de consulta mundial, de datos multidisciplinarias relacionadas con ergonomía, salud ocupacional y DME, que podrían abordar el tema y la población de estudio, las cuales fueron Ovid SP, EBSCO HOST, PROQUEST, MEDLINE, Science Direct, PubMed, y otros motores de búsqueda. La revisión se realizó en el periodo comprendido entre el 2005 - 2015.

La selección (inclusión y rechazo) de la información obedeció a los siguientes criterios:

\section{Criterios de inclusión:}

- Artículos en español o inglés

- Artículos publicados entre el 2005 al 2015

- Artículos relacionados con ergonomía, salud ocupacional y desórdenes musculoesqueléticos.

- Revisiones de información secundaria de instituciones vinculadas con los procesos de rehabilitación.

- Población trabajadora de cualquier sector, sana o con DME

- Modelos de salud implementados para la intervención de los DME

Criterios de exclusión:

- Modelos de rehabilitación en población pediátrica con discapacidad

- Modelos de rehabilitación que busquen la inclusión laboral en personas en condición de discapacidad.

Para la selección de la información se realizó una base de datos que incluyó las variables: título, nombre de la revista, año, publicación, institución o universidad, autores, objetivos, métodos, diseño, resultados $\mathrm{y}$ conclusiones. Con base en lo anterior se realizó la caracterización de los modelos de acuerdo con enfoques, niveles de atención y limitaciones, con el fin de identificar sus particularidades para los procesos de rehabilitación en la población trabajadora.

\section{RESULTADOS}

A partir de la búsqueda se seleccionaron 72 artículos que fueron sometidos a los criterios de inclusión y de exclusión definidos para este estudio de los cuales 60 documentos fueron tenidos en cuenta para el análisis bibliométrico. La Figura 1 representa el proceso seguido para la selección.

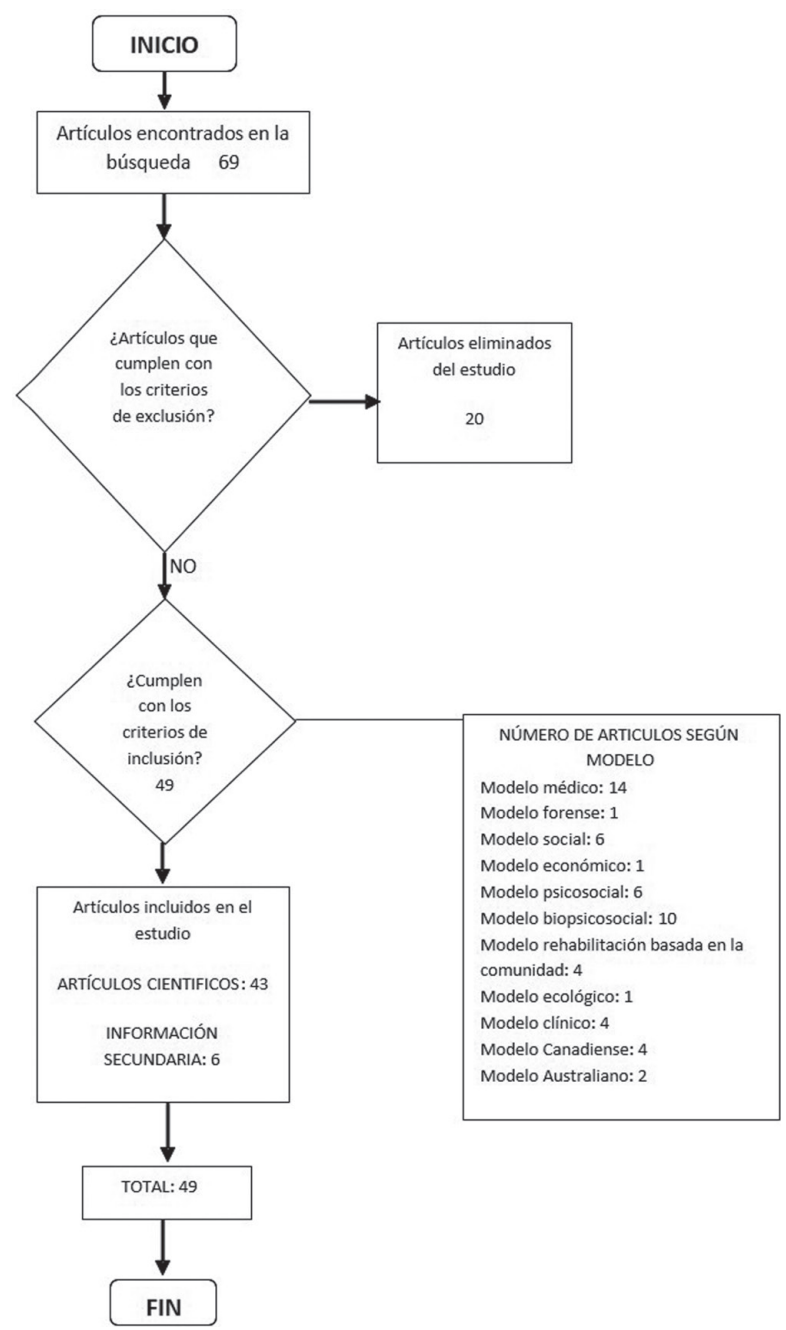

FIGURA 1. Flujograma de los documentos incluidos en la revisión

De los artículos incluidos en la presente revisión se identificaron y abordaron doce modelos teóricos del proceso salud enfermedad, los cuales mostraron un enfoque en rehabilitación clínica. Cada uno de los modelos revisados expone las perspectivas y fundamentos propios que promueven programas para mejorar la calidad de vida y la funcionalidad física y mental de los trabajadores. Los modelos encontrados fueron: modelo médico, forense, social, económico, 
psicosocial, biopsicosocial, rehabilitación basada en la comunidad, ecológico, modelo de servicios de soporte, modelo canadiense, modelo australiano y modelo clínico, algunos caracterizados por tener como fin la inclusión y la reincorporación del individuo al trabajo.

Se identificó que el modelo médico cuenta con mayor evidencia en la literatura científica, ya que se constituye como el punto de partida para el desarrollo de otros modelos en salud y rehabilitación. La mayor frecuencia de citación de los modelos, se refleja en los modelos médico, con 10 citas, seguido por el modelo biopsicosocial con ocho citas. Ver Figura 1. Flujograma de los documentos incluidos en la revisión.
Frente a la temática consultada, la evidencia documental correspondió en un $88 \%$ a artículos de evidencia científica y en un $12 \%$ a información secundaria que contempló capítulos de libros así como algunos documentos del marco de referencia normativo en Colombia. Se encontró con mayor frecuencia en la búsqueda analizada artículos de revisión de la literatura en un $42 \%$, seguido por artículos científicos de corte transversal en un $30 \%$, y un $4 \%$ para ensayo clínico aleatorizado y revisión sistemática. De igual manera se analizaron seis documentos de concepto de expertos, cuatro tesis y una serie de casos.

A continuación se presenta de cada Modelo de rehabilitación encontrado, una breve descripción del enfoque a partir de las distintas referencias seleccionadas. Tabla 1. Modelos de rehabilitación.

TABLA 1. Modelos de rehabilitación

\begin{tabular}{|c|c|c|}
\hline MODELO & AUTOR-AÑO & ENFOQUE \\
\hline \multirow{8}{*}{ Médico } & $\begin{array}{l}\text { Gouttebarge V et al. } 2006^{37} \text {, } \\
\text { Schultz I et al. } 2007^{3} \\
\text { Guzman J. } 2009^{50}, \text { Korhan O } \\
\text { et al. } 2010^{28} \\
\text { Lakke S et al. } 2013^{38} \text {,Herr } \\
\text { R. } 2015^{48}\end{array}$ & $\begin{array}{l}\text { Destaca las modalidades de evaluación fisioterapéutica centradas en pruebas } \\
\text { de capacidad funcional y tratamiento físico en dolor de cuello, espalda, } \\
\text { hombros, muñecas, rodillas y pies, al igual que intervenciones adecuadas } \\
\text { en el puesto de trabajo, dado el gran número de población trabajadora } \\
\text { afectada. }{ }^{37,3,50,28,38,48}\end{array}$ \\
\hline & $\begin{array}{l}\text { Leerar P et al. } 2007^{36}, \text { Korhan } \\
\qquad \text { O et al. } 2010^{28}\end{array}$ & $\begin{array}{l}\text { Su enfoque parte de la detección de la patología orgánica. Los síntomas y } \\
\text { la discapacidad son directamente asociados y proporcionales a la patología } \\
\text { física. }^{36,28}\end{array}$ \\
\hline & $\begin{array}{l}\text { Loisel P et } \\
\text { al. } 200^{55} \text {,Lanfranchi J. } 2008^{57}\end{array}$ & $\begin{array}{l}\text { Destina la intervención en la comprensión de la enfermedad y la discapacidad, } \\
\text { asociada a problemas de salud musculoesquelética. Facilita el desarrollo de } \\
\text { nuevas intervenciones en patologías dentro del campo laboral, para reducir } \\
\text { el ausentismo. }\end{array}$ \\
\hline & Waddell et al. $2009^{7}$ & \\
\hline & Waddell et al. $2009^{7}$ & $\begin{array}{l}\text { A través de este modelo, la asistencia sanitaria y la seguridad en el trabajo } \\
\text { permiten identificar, evaluar y controlar riesgos con el fin de agilizar el } \\
\text { retorno al trabajo. La intervención es de carácter individual. }{ }^{7}\end{array}$ \\
\hline & McColl M et al. $2008^{24}$ & $\begin{array}{l}\text { Considera este modelo como el más empleado para la integración de los } \\
\text { servicios de rehabilitación y de atención primaria. }{ }^{24}\end{array}$ \\
\hline & Koltan A. $2009^{51}$ & $\begin{array}{l}\text { Desarrolla diseños ergonómicos para la población trabajadora, con el fin de } \\
\text { corregir el manejo manual de cargas, siendo este el factor principal de dolor } \\
\text { de espalda baja. }{ }^{51}\end{array}$ \\
\hline & Templeman K et al. $2011^{27}$ & $\begin{array}{l}\text { Integra la medicina complementaria, alternativa y convencional en la } \\
\text { atención primaria de salud. }{ }^{27}\end{array}$ \\
\hline Forense & Schultz I et al. $200^{73}$ & $\begin{array}{l}\text { Evoluciona a partir de la psicología forense y de la investigación } \\
\text { neuropsicológica para explicar las motivaciones del trabajador que podrían } \\
\text { influir en la decisión del retorno al trabajo (simulación, ganancias primarias, o } \\
\text { ganancias secundarias) después de presentarse una alteración en la condición } \\
\text { de salud. }\end{array}$ \\
\hline
\end{tabular}




\section{MODELO \\ AUTOR-AÑO \\ ENFOQUE}

Céspedes G.2005 ${ }^{30}$, Waddel, Destaca la importancia del individuo dentro de la sociedad al reconocer sus

G et al. $2009^{7}$ necesidades, perspectivas y derechos..$^{30,7}$

Colombia, Ministerio de Defensa Nacional, et al. $2009^{16}$, Santos F et al. $2010^{33}$

Social
Busca contribuir a la inclusión social de las personas con discapacidad, a través de la rehabilitación integral. El trabajador hace parte del capital social, humano y productivo del país. Orienta la toma de decisión política, la gestión social y el posicionamiento de corresponsabilidad del Estado. ${ }^{16,33}$ Parte desde el enfoque de una rehabilitación integral más que funcional. ${ }^{16}$

Incluye acciones simultáneas en: promoción de la salud y prevención de Cifuentes $\mathrm{C}$ et al. 2010. ${ }^{41}$ la discapacidad; desarrollo, recuperación y mantenimiento funcional, y la integración socio-ocupacional. ${ }^{41}$

Busca la integración por medio de la rehabilitación ocupacional, de los trabajadores con discapacidad. ${ }^{43}$

Económico

Waddell, G et al. $2009^{7}$

En este modelo se hace evidente la relación entre las tasas de desempleo y la discapacidad en donde la enfermedad conlleva a: privación del empleo, desventaja social y aumento de pobreza. $^{7}$

Busca por medio de la comunicación activa de médicos con los empleadores,

Sullivan MJ et al. 200554, al igual que discutir temas de trabajo directamente con el trabajador que Costa-Black K et al. $2010^{34}$ permitan disminuir los factores de riesgo, así como un seguro y estable retorno al trabajo. ${ }^{34,54}$

Buck R et al. $2011^{35}$

Examina las actitudes y problemas más comunes de los trabajadores, con el fin de crear posibles soluciones. ${ }^{35}$

Psicosocial

Arends I et al. $2012^{52}$,Ahlstrom L et al. $2013^{26}$

Destaca la importancia de la integración de la rehabilitación laboral y las condiciones de apoyo en el trabajo, con el fin de aumentar la capacidad laboral y mejorar el proceso de retorno al mismo. . $^{52,26}$

Colombia, Dirección general de riesgos profesionales. 2015..$^{39}$

El trabajador es sujeto activo de su proceso de rehabilitación junto con su familia, la comunidad laboral y social. Busca la reincorporación y el desarrollo de una actividad ocupacional satisfactoria que lo conduzca a una mejor calidad de vida. ${ }^{39}$

Koltan A. $2009^{51}$, Meyers, A. Identifica los factores de riesgo físicos, biomecánicos, psicológicos, psicosociales y organizacionales, con el fin de establecer programas de

$2010^{47}$, Roman D. $2013^{56}$ prevención de lesiones músculo-esqueléticas relacionadas con el trabajo y evalúa la efectividad del mismo. ${ }^{51,47,56}$

Santos H et al $2012^{42}$, Speerin $\mathrm{R}$ et al. $2011^{31}$

Shaw WS et al $2011^{32}$

SO,Chun Lung, 2011 Major M et al. 2015

Pontonnier C. $2014^{49}$

Major $\mathrm{M}$ et al. $2015^{10}$

Boubaker K et al. 2014
Otorga importancia a la prevención primaria y secundaria; a la coordinación de la atención y el acceso a un equipo multidisciplinario, para el abordaje de DME. $^{42,31}$

Incluye estrategias para evaluar factores ocupacionales que faciliten el retorno al trabajo teniendo en cuenta la percepción del trabajador y del empleador para la modificación del entorno y de la actividad laboral. ${ }^{32}$

Destaca la importancia de los factores clínicos, la percepción del trabajador, el puesto de trabajo y las políticas de organización, con el fin de identificar obstáculos que pueden ser modificables a la hora de retornar el trabajador a sus actividades. ${ }^{45,49,10}$

Da importancia a la reevaluación en términos de riesgo, tiempos y dolor con el propósito de adaptar las tareas y puestos de trabajo. ${ }^{55}$ 
Céspedes G. $2005^{30}$

Rehabilitación $\quad$ McColl M et al. $2009^{21}$
Basada en la
Comunidad
(RBC)

Climent J et al. 2011

Colombia, Ministerio de Salud y de Protección social. $2012^{40}$

Ecológico Carrión F et al. $2011^{5}$

Aiken A. $2007^{44}$, Carrión F et al. $2011^{5}$

Clínico

Mahmud N et al. $2010^{13}$

Heijbela B et al. $2012^{29}$

Davis S. $2006^{18}$

Aristizábal M. $2015^{43}$

\section{Canadiense}

Waddell, G et al. $2009^{7}$

Bidassie B. $2010^{46}$
La participación política y su influencia en temas de discapacidad, ocasiona que haya o no un buen manejo de la enfermedad. ${ }^{30}$

Ha sido diseñada para ofrecer servicios de rehabilitación, con mayor prevalencia en países en desarrollo, teniendo en cuenta que los recursos materiales y humanos son escasos. Considera que los profesionales de la rehabilitación deben movilizar recursos comunitarios frente a las personas con enfermedades crónicas y en situación de discapacidad. ${ }^{21}$

Propone un modelo descentralizado, en donde la fisioterapia es la encargada de la asistencia de las personas con alguna patología o discapacidad, dadas las necesidades actuales de aplicabilidad y de difusión social, con el propósito de mejorar la calidad de vida. ${ }^{4}$

Es una estrategia de desarrollo comunitario para la rehabilitación, se enfoca en el goce efectivo de los derechos humanos. ${ }^{40}$

Resulta de la interacción del agente, huésped y ambiente. Se desarrolla en 4 componentes: proceso, contexto, persona y tiempo. Considera que el individuo es influenciado por el micro, meso y macro sistema. ${ }^{5}$

Caracterizado por la participación de diferentes disciplinas en rehabilitación, en donde cada profesional interviene de forma independiente pero secuencial. ${ }^{44,5}$

Considera que la evaluación de la capacidad funcional para el retorno al trabajo es efectiva en la prevención de nuevas lesiones ocupacionales. ${ }^{13}$

Facilita el reintegro laboral a través de rehabilitación multimodal y vocacional, en trabajadores menores de 55 años con DME y stress. ${ }^{29}$

Contempla los componentes físicos, cognitivos, espirituales y afectivos, y la manera cómo influyen en la ocupación. ${ }^{18}$

Busca la integración por medio de la rehabilitación ocupacional de los trabajadores con discapacidad que han sufrido un accidente de trabajo o presentan una enfermedad de origen laboral..$^{43}$

Se enfoca en la persona en situación de discapacidad y muestra su relación con el entorno y las tareas realizadas que desarrollan desorden musculoesquelético. ${ }^{7}$

Contempla la persona, el entorno y la ocupación, su objetivo es detectar DME y actuar sobre los factores de riesgo, con el fin de mejorar la calidad de vida del trabajador. ${ }^{46}$
A partir de los documentos revisados, se establecieron las características de cada uno de los modelos, sus componentes, los niveles de atención para los que se encuentran diseñados y las limitaciones descritas. Tabla 2. Características de los modelos de rehabilitación. 
Modelos de rehabilitación para la intervención del desorden músculo-esquelético en trabajadores

TABLA 2. Características de los modelos de rehabilitación

\begin{tabular}{|c|c|c|c|}
\hline Modelo & Componentes & $\begin{array}{l}\text { Niveles de } \\
\text { atención }\end{array}$ & Limitaciones \\
\hline \multirow{3}{*}{ Médico } & Reconoce signos y síntomas. ${ }^{4}$ & Secundario & $\begin{array}{l}\text { No vincula contextos como la familia, el } \\
\text { trabajo ni la comunidad. Centrado en la } \\
\text { condición física excluye aspectos mentales, } \\
\text { sociales y culturales. }\end{array}$ \\
\hline & Identifica la causa biológica de la patología. ${ }^{4}$ & & \\
\hline & $\begin{array}{l}\text { El trabajo es considerado un riesgo potencial } \\
\text { para la salud. } .^{58}\end{array}$ & Terciario & $\begin{array}{l}\text { No permite divisar los múltiples factores } \\
\text { que pueden ser causantes de la enfermedad. }\end{array}$ \\
\hline Forense & $\begin{array}{l}\text { Explica las interacciones, conocimientos y } \\
\text { motivaciones de la persona lesionada } \\
\text { Suele utilizar un modelo interdisciplinario. }{ }^{3}\end{array}$ & Terciario $^{3}$ & $\begin{array}{l}\text { Centrado en el razonamiento de la } \\
\text { medicación y el manejo efectivo de los } \\
\text { casos más que la determinación de la } \\
\text { relación causal. }\end{array}$ \\
\hline Social & $\begin{array}{l}\text { Se centra en el reconocimiento de los } \\
\text { derechos y necesidades de las personas en } \\
\text { condición de discapacidad. }{ }^{7}\end{array}$ & Terciario & $\begin{array}{l}\text { Desconoce las influencias psicológicas y } \\
\text { personales sobre la discapacidad. }{ }^{7}\end{array}$ \\
\hline Económico & $\begin{array}{l}\text { Describe el impacto de los beneficios } \\
\text { financieros en el comportamiento de la } \\
\text { enfermedad. }{ }^{7,30}\end{array}$ & Terciario & $\begin{array}{l}\text { La condición de salud individual pasa a un } \\
\text { segundo plano. }{ }^{3}\end{array}$ \\
\hline Psicosocial & $\begin{array}{l}\text { Posee una perspectiva cognitivo-conductual. } \\
\text { Usa un modelo interdisciplinario. }{ }^{3}\end{array}$ & Terciario & $\begin{array}{l}\text { Da mayor importancia a las percepciones, } \\
\text { creencias y expectativas del individuo. }{ }^{3}\end{array}$ \\
\hline Biopsicosocial & $\begin{array}{l}\text { Busca explicar la condición de discapacidad } \\
\text { y el retorno al trabajo a partir de la } \\
\text { comprensión del individuo, los sistemas } \\
\text { involucrados y sus interacciones. }{ }^{3}\end{array}$ & Primario & $\begin{array}{l}\text { Falta mayor integración con elementos } \\
\text { ambientales y sociales. Orientado por el } \\
\text { establecimiento de factores de riesgo. }{ }^{31}\end{array}$ \\
\hline $\begin{array}{l}\text { Rehabilitación } \\
\text { basada en comunidad }\end{array}$ & $\begin{array}{l}\text { Es considerada por algunos autores más } \\
\text { que un modelo como una estrategia } \\
\text { descentralizada que ofrece servicios de } \\
\text { rehabilitación. }{ }^{30,59}\end{array}$ & Primario & $\begin{array}{l}\text { Se centra en resolver problemas de } \\
\text { integración social. }{ }^{7}\end{array}$ \\
\hline Ecológico & $\begin{array}{l}\text { Contempla el proceso, el contexto, la } \\
\text { persona y el tiempo. Analiza la influencia en } \\
\text { el individuo, con la comunidad más cercana } \\
\text { y con los patrones culturales, tendencias } \\
\text { políticas, sistemas económicos y creencias. }\end{array}$ & Secundario & $\begin{array}{l}\text { No contempla factores protectores para } \\
\text { prevenir alteraciones en la condición de } \\
\text { salud del trabajador. }\end{array}$ \\
\hline Canadiense & $\begin{array}{l}\text { Contempla componentes físicos, cognitivos, } \\
\text { afectivos y espirituales. }{ }^{18}\end{array}$ & $\begin{array}{c}\text { Primario } \\
\text { Secundario }\end{array}$ & $\begin{array}{l}\text { Se centra en la rehabilitación dirigida a la } \\
\text { participación del individuo en la actividad. }{ }^{18}\end{array}$ \\
\hline Australiano & $\begin{array}{l}\text { Va dirigido a la intervención temprana y } \\
\text { rehabilitación ocupacional. Empodera a los } \\
\text { empresarios. }{ }^{12}\end{array}$ & Primario & $\begin{array}{l}\text { Está centrado en el marco político y legal } \\
\text { Australiano. }\end{array}$ \\
\hline Clínico & $\begin{array}{l}\text { Considera a la enfermedad como una falla } \\
\text { ocasionada por un daño biológico. } .^{5}\end{array}$ & Secundario & $\begin{array}{l}\text { El diagnóstico provee una información } \\
\text { limitada acerca de la condición de } \\
\text { discapacidad, ya que desconoce los factores } \\
\text { socioculturales y no contemplan las } \\
\text { experiencias subjetivas del paciente. }\end{array}$ \\
\hline
\end{tabular}

Dentro de la revisión realizada se encontraron otras categorías de modelos en rehabilitación, las cuales parten de la forma en la que se gestionan los recursos para la prestación de éstos servicios; un ejemplo de ello es el modelo de servicios de soporte el cual basa su enfoque en la enfermedad y a partir de esta plantea como se proporciona el apoyo terapéutico y los equipos que se requieren para su intervención. Opera a través 
de organizaciones comunitarias no gubernamentales y profesionales que desarrollan su actividad para la comunidad, fomentando la accesibilidad con el fin de lograr a cabalidad el proceso de rehabilitación ${ }^{5}$.

\section{DISCUSIÓN}

Los modelos de salud que integran enfoques de rehabilitación establecen una cercanía a los problemas reales de los trabajadores al considerar la comprensión intelectual y emocional de la naturaleza del problema ${ }^{9,10}$, y no solamente desde un concepto biologista.

El manejo de los DME de origen laboral ha sido abordado por el sector salud y su objetivo se ha focalizado predominantemente hacia acciones para el control y alivio de los síntomas. Sin embargo, la condición de salud del trabajador va más allá, y debe ser abordada a partir de los diferentes determinantes que influyen en ella, sin dejar de lado el papel del trabajador y el liderazgo de los empleadores en el fomento de acciones orientadas a la salud en el ambiente productivo ${ }^{7,11,12}$.

Mahmud, et al. señalan la importancia de incluir en el modelo de rehabilitación el paradigma de tratamiento y el paradigma de prevención basado en un modelo biopsicosocial, el cual busca evitar la evolución de la condición de salud hacia la discapacidad prolongada del trabajador, considerando y haciendo efectivas las modificaciones en los lugares de trabajo ${ }^{13,14}$.

Frente al modelo biopsicosocial existe poca evidencia científica acerca de su efectividad en procesos de rehabilitación en DME ocupacionales que generan por ejemplo dolor de cuello y hombro ${ }^{15}$. Este modelo aunque es comúnmente utilizado no cuenta con estudios experimentales que permitan determinar el impacto de la implementación de este modelo de rehabilitación en la capacidad funcional laboral del individuo.

Se destaca en los modelos Social y Psicosocial, acciones de tipo legal y normativo facilitadas por entes políticos hacia la población trabajadora en condición de discapacidad, a través del desarrollo de un sistema de rehabilitación integral y la construcción de Centros de Rehabilitación que faciliten el análisis, direccionamiento, y guía para la aplicación de una política sectorial de discapacidad y el cambio de una rehabilitación funcional a una rehabilitación integral sostenible para dicha población ${ }^{16,17}$.

Otros modelos como el canadiense ${ }^{18}$ incluyen la espiritualidad como esa singularidad del individuo, independiente de sus capacidades que va más allá de los aspectos físicos, afectivos y cognitivos. Por lo anterior los procesos de rehabilitación no sólo se enfocan en la condición de discapacidad sino en la persona que los sufre. Este modelo da importancia a los valores, creencias y objetivos significativos para cada individuo que se convierten de vital importancia a la hora de definir las restricciones en la participación y en el reintegro o reubicación de un trabajador.

Cabe destacar en el modelo australiano ${ }^{12}$, el enfoque hacia una intervención anticipada a través de la identificación de signos de alerta temprana desde lo físico, lo fisiológico y lo comportamental, siendo de gran ayuda para los empleadores en la búsqueda de estrategias que ayuden a los trabajadores que se encuentran en riesgo de lesiones. Este modelo se gestiona a partir de la cooperación voluntaria de todos los involucrados en el proceso.

Modelos como el de RBC y de servicios de soporte son considerados en algunos casos estrategias para abordar las problemáticas de salud. El modelo de RBC tiene en cuenta aspectos económicos y sociales propios de la población y el modelo de servicios de soporte brinda la atención a partir de los recursos del sistema de salud ${ }^{5}$. Sin embargo, un modelo de rehabilitación debería contemplar las necesidades tanto del microsistema, mesosistema y el macrosistema.

Si bien es cierto que cada uno de los modelos revisados busca el cuidado y tratamiento de una condición de salud, sólo a partir del modelo biopsicosocial se explica la discapacidad y el retorno al trabajo a través de la compresión del individuo, los sistemas involucrados y sus interacciones ${ }^{3}$.

En relación con lo anterior, la intervención a través de modelos como el propuesto por la CIF, permiten no solo el análisis y la comprensión de problemas clínicos, sino integrar en el abordaje de la discapacidad el entorno del individuo. De igual manera, Delisa propone incluir un enfoque interdisciplinario para la rehabilitación en investigación ${ }^{19}$. Así mismo, autores como Seel, et al. presentan modelos de enfoques científicos de predicción en las poblaciones de rehabilitación ${ }^{20}$.

La revisión realizada determinó que no existen conflictos entre modelos, se observa que los elementos, herramientas y niveles de atención son complementarios y la tendencia es validar una práctica dirigida ${ }^{2}$. El conocimiento de distintos modelos de salud y de rehabilitación junto con los procesos y procedimientos 
permite concluir la importancia de realizar un tránsito desde lo disciplinar a lo transdisciplinar que favorezca la intervención oportuna del trabajador con DME, a través de un abordaje entorno a la promoción, prevención y asistencia oportuna ${ }^{2,3,21,22}$.

La intervención para los trabajadores con DME se basa tradicionalmente en el modelo biomédico de la salud, que comprende el reconocimiento de signos y síntomas, la realización de un diagnóstico y un plan de tratamiento y/o rehabilitación para restaurar la función para el retorno a su actividad laboral. Sin embargo no se puede desconocer que el DME es de origen multifactorial y que no solo la identificación y el manejo de una deficiencia estructural y funcional deben ser consideradas en los procesos de rehabilitación.

En el mismo sentido, a través del tiempo se ha trabajado en la descentralización de la asistencia rehabilitadora, poniendo de manifiesto un modelo de rehabilitación que abandona la enfermedad, para centrarse primero en el paciente, su familia, para terminar enfocándose en la comunidad, lo anterior en busca de una visión integral del paciente en todas sus necesidades para darle una atención de calidad, basada en la evidencia científica pero ajustada a cada individuo ${ }^{23}$.

La evidencia científica desconoce la efectividad de los modelos de rehabilitación, lo cual puede deberse a la pobre articulación entre la implementación del modelo a un sistema de gestión que permita determinar la efectividad en la intervención de los DME en esta población. Sin embargo se ha establecido que dependiendo del grupo social y las características personales del trabajador un modelo puede tener mayor efectividad que otro.

Por otra parte estudios realizados previamente se centran únicamente en factores de riesgos ocupacionales o en estilos de vida ${ }^{58,59}$ asociados al DME, pero no consideran modelos para el estudio de las condiciones de salud crónicas ${ }^{60}$.

Por lo anterior se hace necesario el desarrollo de modelos transdisciplinarios, que aborden aspectos temporales y multidimensionales para la prevención y la intervención de la condición de discapacidad secundaria al DME de origen laboral que vayan más allá de la atención de la condición de salud ${ }^{3}$. Para lo cual es de vital importancia el desarrollo de una formación a nivel postgradual en los profesionales que interactúan en los procesos de rehabilitación de los trabajadores, con el propósito de desarrollar investigaciones que demuestren el impacto de los modelos de rehabilitación así como la creación de modelos predictores que permitan la prevención del DME.

\section{REFERENCIAS}

1. Piña B. Modelos de explicación sobre la determinación del proceso Salud-Enfermedad. Factores de riesgo en la comunidad; Tomo I. México. D.F: Universidad Autónoma de México; 1990.

2. Schultz I, Stowell A, Feuerstein M, Gatchel RJ. Models of return to work for musculoskeletal disorders. J Occup Rehabil. 2007; 17(2): 327-352. DOI: 10.1007/s10926-007-9071-6.

3. Arredondo A. Análisis y reflexión sobre los modelos teóricos del proceso salud enfermedad. Cad Saúde Públ. 1992; 8(3): 254-261. DOI: http://dx.doi. org/10.1590/S0102-311X1992000300005.

4. Climent JM, Rodriguez Ruiz C, Mondejar F. Evolución histórica de la asistencia de rehabilitación: desde un modelo hospitalario a un modelo descentralizado. Rehabilitación. 2011; 45(1): 2-7. DOI: 10.1016/S0048-7120(11)70002-0.

5. Carrión-Pérez F, López-Medina S, RodríguezMoreno S. Modelos de rehabilitación en atención primaria y comunitaria Revisión bibliográfica. Rehabilitación. 2011; 45(Supl 1): 8-13. DOI: 10.1016/S0048-7120(11)70003-2.

6. Siegert R, McPherson K, Dean S. Theory development and a science of rehabilitation. Disabil Rehabil. 2005; 27(24): 1493-1501. DOI: 10.1080/09638280500288401.

7. Waddell G, Aylward M. Models of sickness and disability applied to common health problems. The Royal Society Of Medicine Press. 2009; 1-64.

8. Moher D, Liberati A, Tetzlaff J, Altman D, PRISMA Group. Preferred reporting items

9. for systematic reviews and meta-analyses: the PRISMA statement. Ann Intern Med. 2009; 151(4): 264-269. DOI: 10.7326/0003-4819-151-4200908180.

10. Geelena R, Soonsb P. Rehabilitation: an 'everyday' motivation model. Patient Educ Couns. 1996; 28(1): 69-77. DOI: 10.1016/0738-3991(96)00871-3.

11. Major M, Vezina N. Analysis of worker strategies: a comprehensive understanding for the prevention of work related musculoskeletal disorders. Int J Ind Ergon. 2015; 48: 149-57. DOI: http://dx.doi.org. ez.urosario.edu.co/10.1016/j.ergon.2015.05.003.

12. Wynne-Jones G, Buck R, Varnava A, Phillips C, Main C. Impacts on work absence and performance: what really matters?. Occup Med (Lond). 2009; 59: 556-562. DOI: $10.1093 /$ occmed/kqp125. 
13. Australian Government. Work Health and Safety Regulations $\mathrm{N}^{\circ} 262$, Act 2011. 2016

14. MahmudN, Schonstein E, Schaafsma F, LehtolaMM, Fassier JB, Verbeek JH, et al. Functional capacity evaluations for the prevention of occupational reinjuries in injured workers. Cochrane Database Syst Rev. 2010. DOI: 10.1002/14651858.CD007290. pub2.

15. Loisel P, Durand M, Berthelette D, Vézina N, Baril R, Gagnon D, et al. Disability prevention new paradigm for the management of occupational back pain. Dis Manage Health Outcomes. 2001; 9 (7): 351-360. DOI: 10.2165/00115677-20010907000001 .

16. Karjalainen K, Malmivaara A, van Tulder M, Roine $\mathrm{R}$, Jauhiainen M, Hurri H, et al. Multidisciplinary biopsychosocial rehabilitation for neck and shoulder pain among working age adults. Cochrane Database Syst Rev. 2010; (3): CDO02194. DOI: 10.1002/14651858.CD002194.

17. Colombia, Ministerio de Defensa Nacional, Ministerio de la Protección Social, Departamento Nacional de Planeación, SENA. Sistema de Rehabilitación integral para la fuerza pública. Documento Conpes 3591. 2009.

18. Holtermann A, Jorgensen MB, Gram B, Christensen JR, Faber A, et al. Worksite interventions for preventing physical deterioration among employees in job-groups with high physical work demands: background, design and conceptual model of FINALE. BMC Public Health. 2010; 10: 1-120. DOI: $10.1186 / 1471-2458-10-120$.

19. Davis S. Rehabilitation: The use of theories and Models in Practice. British: Elsevier Health Sciences; 2006.

20. Delisa JA. Shaping the future of medical rehabilitation research: using the interdisciplinary research model. Arch Phys Med Rehabil. 2004; 85(4): 531-537. DOI: 10.1016/j.apmr.2003.12.003.

21. Seel R, Steyerberg E, Malec J, Sherer M, Macciocchi SN. Developing and evaluating prediction models in rehabilitation populations. Arch Phys Med Rehabil. 2012; 93(Supp18): 138-53. DOI: 10.1016/j. apmr.2012.04.021.

22. McColl M, Shortt S, Godwin M, Smith K, Rowe K, O'Brien $\mathrm{P}$, et al. Models for integrating rehabilitation and primary care: a scoping study. Arch Phys Med Rehabil. 2009; 90(9): 1523-1531. Doi: 10.1016/j. apmr.2009.03.017.

23. Klimenko E, Julliard K, Lu S, Song H. Models of health: a survey of practitioners. Complement Ther Clin Pract. 2006; 12(4): 258-267. DOI: 10.1016/j. ctcp.2006.05.003.
24. Evanoff B, Dale AM, Descatha A. A conceptual model of musculoskeletal disorders for occupational health practitioners. Int $\mathrm{J}$ Occup Med Environ Health. 2014; 27(1): 145-148. DOI: 10.2478/s13382-014-0232-5.

25. Ahlstrom L, Hagberg M, Dellve L. Workplace Rehabilitation and Supportive Conditions at Work: A Prospective Study. J Occup Rehabil. 2013; 23(2): 248-260. DOI: 10.1007/s10926-012-9391-z.

26. Templeman $\mathrm{K}$, Robinson A. Integrative medicine models in contemporary primary health care. Complement Ther Med. 2011; 19(2): 84-92. DOI: 10.1016/j.ctim.2011.02.003.

27. Korhan O, Mackieh A. A model for occupational injury risk assessment of musculoskeletal discomfort and their frequencies in computer users. Saf Science. 2010; 48(7): 868-877. DOI: http://dx.doi. org.ez.urosario.edu.co/10.1016/j.ssci.2010.03.010.

28. Heijbel B, Josephson M, Vingård E. Implementation of a rehabilitation model for employees on long-term sick leave in the public sector: difficulties, countermeasures and outcomes. Work. 2013; 45(3): 323333. DOI: $10.3233 /$ wor-2012-1478.

29. Cespedes G. La nueva cultura de la discapacidad y los modelos de rehabilitación. Rev Aquichan. 2005; 1(5): 108-13.

30. Speerin R, Slater H, Li L, Moore K, Chan M, Dreinhöfer $\mathrm{K}$, et al. Moving from evidence to practice: Models of care for the prevention and management of musculoskeletal conditions. Best Pract Res Clin Rheumatol. 2014; 28(3): 479-515. DOI: 10.1016/j.berh.2014.07.001.

31. Shaw WS, Main CJ, Johnston V. Addressing occupational factors in the management of low back pain: implications for physical therapist practice. Phys ther. 2011; 777-789. DOI: 10.2522/ ptj.20100263.

32. Santos F, Dávila A, Serrato MA. Vicepresidencia de la República, Programa Presidencial para la Acción Integral contra Minas Antipersonal (PAICMA). Modelo de Rehabilitación Integral Inclusiva MRII. De la asistencia a la inclusión social. 2010.

33. Costa-Black K, Loisel P, Anema J, Pransky G. Back pain and work. Best Pract Res Clin Rheumatol. 2010; 24: 227-240. DOI: http://dx.doi.org/10.1016/j. berh.2009.11.007.

34. Buck R, Porteous C, Wynne-Jones G, Marsh K, Phillips C, Main C. Challenges to remaining at work with common health problems: what helps and what influence do organisational policies have? J Occup Rehabil. 2011; 21(4): 501-512. DOI: 10.1007/ s10926-011-9288-2.

35. Leerar PJ, Boissonnautt W, Domholdt E, Roddey T. 
Documentation of Red Flags by physical therapists for patients with low back pain. J Man Manip Ther. 2007; 15: 42-49. DOI: 10.1179/jmt.2007.15.2.42E.

36. Gouttebarge V, Wind H, Kuijer PP, Sluiter JK, Frings-Dresen MH. Reliability and agreement of 5 Ergo-Kit functional capacity evaluation lifting tests in subjects with low back pain. Arch Phys Med Rehabil. 2006; 87(10): 1365-1370. DOI: 10.1016/j. apmr.2006.05.028.

37. Lakke SE, Soer R, Geertzen J, Wittink H, Douma $\mathrm{R}$, van der Schans $\mathrm{C}$ et al. Construct validity of functional capacity tests in healthy workers. BMC Musculoskeletal Disorders. 2013; 14: 180. DOI: 10.1186/1471-2474-14-180.

38. República de Colombia, Ministerio de la protección social. Dirección General de Riesgos profesionales. Manual de procedimientos para la rehabilitación y reincorporación ocupacional de los trabajadores en el sistema general de riesgos profesionales. 2010.

39. Ministerio de Salud. Lineamientos Nacionales de Rehabilitación basada en la comunidad - RBC. 2014.

40. Ministerio de la protección social. Manual Guía sobre procedimientos para la rehabilitación y reincorporación ocupacional de los trabajadores en el sistema general de riesgos profesionales. 2004.

41. Santos H, Martínez S, Castro V. Worker's Health'Program in the Institute of Social Security of Tabasco State, México. 2010. Rev Cuban Salud Trabajo 2012; 13(1): 41-7.

42. República de Colombia, Ministerio de la protección social. Dirección General de Riesgos profesionales. Manual Guía de Procedimientos. Instrumentación del Manual Guía de Procedimientos de Rehabilitación y Reincorporación Ocupacional de los Trabajadores en el Sistema General de Riesgos Profesionales. 2004.

43. Aiken A. The design, implementation, and evaluation of a model of collaborative care to improve treatment options for people with musculoskeletal impairments in Ontario. Queen's University (Canada), 2007; 1:194.

44. So BCL, Hung L. Development of ErgoCoach Model (Participatory Ergonomics) to prevent work-related musculoskeletal disorders among aircraft cabin cleaners. Paper presented in the 30th Annual Congress of The Hong Kong Orthopaedic Association. Orthopaedics \& Traumatology 2010; 45.

45. Bidassie B. Development of a predictive model for low back musculoskeletal disorders based on occupational and lifestyle risk factors. Purdue University. 2010.
46. Meyers A. Prevention strategies for musculoskeletal disorders among high-risk occupational groups. Thesis, University of Iowa, 2010.

47. Herr R, Bosch J, Loerbroks A, Vianen A, Jarczoka M, Fischer J, et al. Three Job Stress Models and Their Relationship with Musculoskeletal Pain in Blue- and White-collar Workers. Journal of Psychosomatic Research. 2015; 79(5): 340-347. DOI: 10.1016/j.jpsychores.2015.08.001.

48. Pontonnier C, Zee M, Samani A, Dumont G, Madeleine P. Strengths and limitations of a musculoskeletal model for an analysis of simulated meat cutting tasks. Appl Ergon. 2014; 45(3): 592600. DOI: 10.1016/j.apergo.2013.08.003.

49. Guzman J, Hurwitz E, Carroll L, Haldeman S, Côté $\mathrm{P}$, Carragee $\mathrm{E}$, et al. A new conceptual model of neck pain: linking onset, sourse, and care: the bone and joint decade 2000-2010 task force on neck pain and Its associated disorders. Spine. 2009; 33( Suppl): S14-23. DOI: 10.1097/BRS.0b013e3181643efb.

50. Koltan A. An ergonomics approach model to prevention of occupational musculoskeletal injuries. Int J Occup Saf Ergon. 2009; 15(1): 113-124. DOI: 10.1080/10803548.2009.11076793.

51. Arends I, Bruinvels DJ, Rebergen DS, Nieuwenhuijsen K, Neumeyer-Grömen A, Madan I, et al. Interventions to facilitate return to work in adults with adjustment disorders. Cochrane Database Syst Rev. 2012; CD006389. DOI: 10.1002/14651858.CD006389.pub2.

52. Loisel P, Buchbinder R, Hazard R, Keller R, Scheel I, Van Tulder M, et al. Prevention of work disability due to musculoskeletal disorders: the challenge of implementing evidence. J Occup Rehabil. 2005; 15(4): 507-524. DOI: 10.1007/s10926-005-8031-2.

53. Sullivan MJ, Ward LC, Tripp D, French DJ, Adams H, Stanish WD. Secondary prevention of work disability: community-based psychosocial intervention for musculoskeletal disorders. J Occup Rehabil. 2005; 15(3): 377-392 DOI: 10.1007/ s10926-005-5944-7.

54. Boubaker K, Colantoni A, Allegrini E, Longo L, Giacinto S, Monarca D et al. A model for musculoskeletal disorder-related fatigue in upper limb manipulation during industrial vegetables sorting. Int J Ind Ergon. Issue 4. 2014; 4(44): 601605. DOI: 10.1016/j.ergon.2014.03.005.

55. Roman-Liu D. External load and the reaction of the musculoskeletal system - A conceptual model of the interaction. Int J Ind Ergon. 2013; 4(43): 356-362. DOI: 10.1016/j.ergon.2013.04.002.

56. Lanfranchi J, Duveau A. Explicative models of musculoskeletal disorders (MSD): From 
biomechanical and psychosocial factors to clinical analysis of ergonomics. Eur Rev Appl Psychol. 2008; 58(4), 201-213. DOI: 10.1016/j.erap.2008.09.004.

57. Domenech J, Sánchez-Zuriaga D, Segura-Ortí E, Espejo-Tort B, Lisón JF. Impact of biomedical and biopsychosocial training sessions on the attitudes, beliefs, and recommendations of health care providers about low back pain: a randomised clinical trial. Pain. 2011; 152(11): 2557-2563. DOI: 10.1016/j.pain.2011.07.023.

58. Briand C, Durand MJ, St-Arnaud L, Corbiére M. Work and mental health: Learning from return-towork rehabilitation programs designed for workers with musculoskeletal disorders. Inter. J. of law and phsychiatry. 2007; 30(4-5): 444-457. DOI: 10.1016/j.ijlp.2007.06.014.

59. Melloh M, Elfering A, Chapple CM, Käser A, Rolli Salathé C, Barz T, et al. Prognostic occupational factors for persistent low back pain in primary care. Int Arch Occup Environ Health. 2013; 86: 261-269. DOI: $10.1007 / \mathrm{s} 00420-012-0761-9$.

60. Ramond A, Bouton C, Richard I, Roquelaure Y, Baufreton C, Legrand E, et al. Psychosocial risk factors for chronic low back pain in primary care--a systematic. Review. Fam Pract. 2011; 28(1): 12-21. DOI: $10.1093 /$ fampra/cmq072.

61. Deyo RA, Mirza SK, Turner JA, Martin BI. Overtreating chronic back pain: time to back off? J Am Board Fam Med. 2009; 22: 62-68. DOI: 10.3122/jabfm.2009.01.080102. 